\title{
Local Heschl's Gyrus-based coordinate system for intersubject comparison of M50 auditory response modeled by single equivalent current dipole
}

\author{
Todor Jordanov*, Tzvetan Popov, Christian Wienbruch, Thomas Elbert, Brigitte Rockstroh \\ Department of Psychology, University of Konstanz, Germany
}

\begin{abstract}
A B S T R A C T
Allocating electromagnetic auditory responses to active regions in the human auditory cortex can be difficult because of high interindividual variability of the relevant structures. Location and orientation of the primary auditory cortex (Heschl's Gyrus) and the temporal plane vary with individual features such as age, gender, handedness, or between healthy subjects and patients with a psychiatric disorder (e.g., schizophrenia). Here, we propose a reference coordinate system that considers the individual MRI-based position, orientation and length of the primary auditory cortex to account for interindividual variability. Transformation of the M50 dipole localizations in this new HG-(Heschl's-Gyrus)-coordinate system, accomplished for 10 healthy subjects and 10 schizophrenia patients, confirmed group difference more precisely than other registration methods. We suggest to use the HG-coordinate system for localization of functional measures and evaluation of brain activity differences between groups or measurement conditions.
\end{abstract}

\section{Introduction}

The comparison of anatomical areas and functional activities between individual human brains encounters the problem of the high interindividual variability of brain structures. Three commonly used categories of methods to deal with this problem include (a) the registration within a global intrinsic coordinate system using, for instance, the anterior and posterior commissures (AC and PC) as reference landmarks, (b) global normalization of individual data with reference to 3D brain volume (e.g., Talairach and Tournoux, 1988; Evans et al., 1993; Friston et al., 1995), or (c) global normalization with respect to gyral and sulcal patterns on the cortical surface (Thompson and Toga, 1996; Van Essen and Drury, 1997; Van Essen et al., 1998; Fischl et al., 1999; Dickson et al., 2001). All of these methods can be used to assign activity obtained from functional magnetic resonance imaging (fMRI), electroencephalography (EEG) and magnetoencephalography (MEG) to anatomical structures. (a) leads to more accurate results for anatomical structures near to the reference points ( $A C$ and $P C$ ) than for structures more distant to the reference points or for structures that differ in localizations between the hemispheres, such as the temporal plane (planum temporale, PT). A major limitation of the volume based registration methods (b) is that the average brain loses its

\footnotetext{
* Corresponding author at: Department of Psychology, University of Konstanz, PO Box 25, D-78457 Konstanz, Germany. Tel. + +49 7531883086

fax: +497531884601 .

E-mail address: todor.iordanov@uni-konstanz.de (T. Jordanov).
}

representative surface structure by obscuring gyral and sulcal organization (Kang et al., 2004). The third group of methods (c) seeks to consider the natural organization of the brain. Given that the neurons of the human cerebral cortex are arranged in a densely folded sheet forming the surface all over the brain the more natural modeling of the cortex should be a surface instead of 3D volume. Although the surface is a good model of the human cerebral cortex it is not necessarily suitable for intersubject comparisons of brain activity modeled by a single equivalent current dipole using volume conductor models with typically three spatial degrees of freedom. Furthermore, the source localization inaccuracy due to the uncertainty pertaining to the inverse algorithm, a low signal to noise ratio and a functional to structural dataset co-registration errors can cause a dipole localization displacement up to few millimeter in an arbitrary direction within the volume conductor model. The projection of that error afflicted dipole localization on the cortical surface can cause an additional error in the brain activity localization. Therefore, the surface-based co-registration methods are more suitable for group comparison of cortical activity using distributed source models rather than single equivalent current dipole models.

One common characteristic of all the methods linked above is that they act in a global manner, i.e. they register entire brains with all sulci and gyri. This can be considered a disadvantage if the goal is to compare structures small in size and variable in position and form such as Heschl's Gyrus (HG). In that case methods based on local landmarks and structures are required. Several approaches have been published to locally compare auditory cortices. Among these are surface-based (Kang et al., 2004), also local parcella- 
tion methods (Kim et al., 2000; Engel et al., 2009), volume based automated HG extraction method (Tahmasebi et al., 2009) and nonlinear volume based registration of individual HG with a standard brain's HG (Viceic et al., 2009). All these methods are useful and precise but they may not be the perfect choice for intersubject comparison of functional activity modeled by single equivalent current dipole due to an error affliction of the dipole localization and the nonlinear error propagation caused by these methods. That is, dipole localizations will always be subject to errors caused by e.g., low signal to noise ratio or uncertainty pertaining to the inverse algorithm. Thus, the dipole fit will likely be displaced from the true source by a certain distance. Nonlinear transformation approaches can augment this inherent localization error even further.

Precise mapping of functional activity is particularly important when aiming to relate regional functional activity to specific group characteristics, to curtail dysfunctional regions when comparing specific patient groups, or to assess functional regional activity between measurements within the same subject, for instance, as a function of intervention.

Here a reference coordinate system is described that should compensate for interindividual variability in the comparison of structural and functional measures between and within subjects. The coordinate system was developed and evaluated for Heschl's Gyrus (HG) as a crucial structure of the primary auditory cortex in the human brain, which is located anterior to the PT and exhibits hemispheric asymmetry. HG is extended more anterior in the right than in the left hemisphere, this asymmetry varying (between individuals) with handedness, gender, or age. Moreover, reduced asymmetry has been reported in schizophrenia patients (Rossi et al., 1992, 1994; Petty et al., 1995; Barta et al., 1997; Hirayasu et al., 2000; Kasai et al., 2003). The proposed coordinate transformation serves to account for this interindividual variance. It combines the simplicity and speed performance of the linear with the high accuracy of the nonlinear registration methods. Its validity was probed in the study of reduced hemispheric asymmetry in schizophrenia patients compared to healthy subjects.

\section{Materials and methods}

\subsection{Subjects}

Structural magnetic resonance images and magnetoencephalographic data obtained from 10 healthy subjects ( 1 female, 23-29 years) and 10 inpatients meeting ICD-10 Classification of Mental and Behavioural Disorders of schizophrenia (F20.0, 1 female, 20-49 years) served for the development and testing of the HG-coordinate system transformation. All subjects were informed about the measurement procedures and gave written consent.

\subsection{Data acquisition}

T1 weighted structural magnetic resonance images (MRI) were obtained from each participant using a Philips Gyroscan 1.5 T magnet resonance imaging system (Philips Medical Systems, Gyroscan ACS-T). The resolution of the MRIs was $256 \times 256 \times 200$ voxels, with 200 slices covering the left-to-right direction. For identification of head orientation a marker was attached on the right cheek of every subject before starting the MRI scan. For further analysis raw data files were converted with the r2aGUI tool V.2.5.1 (Medical Center and Helmholtz Institute, Utrecht University, Netherlands) in Neuroimaging Informatics Technology Initiative (NIfTI) format.

Functional activity of the auditory cortex was measured using magnetoencephalography (MEG). MEG was recorded while the subject was lying in a supine position, using a 148-channel magnetometer (MAGNESTM 2500 WH, 4D Neuroimaging, San Diego, USA) inside a magnetically shielded room (MSR, Vacuumschmelze,
Hanau). Data were continuously recorded with a sampling rate of $678.17 \mathrm{~Hz}$ and a real bandpass filter of 0.1 to $200 \mathrm{~Hz}$. For artifact control, the vertical and horizontal electrooculogram (EOG) was recorded from four electrodes placed near the left and right temporal canthus and above and below the right eye using a SynAmps amplifier (NEUROSCAN Laboratories, Sterling, VA, USA). The subject's nasion, left and right ear canal, and headshape were digitized with a Polhemus 3Space ${ }^{\circledR}$ Fasttrack prior to measurement. Subjects passively listened to pairs of clicks following the standard pairedclick design for the assessment of M50 (Adler et al., 1982): 100 pairs of $3 \mathrm{~ms}$ square-wave clicks were presented with a $500 \mathrm{~ms}$ offsetto-onset interval and an $8 \mathrm{~s}$ jittered inter-trial interval (offset to onset). Clicks were presented at $50 \mathrm{~dB}$ above the subjective hearing level, which was determined separately for each ear, and delivered via plastic tubes to the subject within the shielded MEG recording chamber. Subjects had no task but were asked to keep their eyes focused on a small fixation point at the ceiling throughout the measurement.

\subsection{Data analysis}

Source localization with Curry Software V.6 (Compumedics Neuroscan USA Ltd.) was based on 68 sensors, 34 over the left and 34 over the right hemisphere, with the aim in mind to exclude magnetic fields generated from non-auditory sources and consequently to increase the goodness of fit for the source localizations. Each 1500-ms epoch (beginning $500 \mathrm{~ms}$ before the first click) was referred to a $100-\mathrm{ms}$ pre-stimulus interval. Epochs were filtered using functions with Gaussian shaped window slopes: high pass frequency $5 \mathrm{~Hz}$ (lower edge frequency width $8 \mathrm{~Hz}$ ) and low pass frequency $55 \mathrm{~Hz}$ (upper edge frequency width $11 \mathrm{~Hz}$ ). Co-registration of the MEG data with the individual MRI comprised two steps: (1) the nasion point and the left and right auricular points (LA and RA) were used for rough alignment; (2) the subject's digitized headshape was aligned to the headshape drawn from the MRI. A three-layer boundary element model (BEM) generated for every subject was served as volume conductor in the dipole fit procedure using two fixed equivalent current dipoles with seed points in the middle of the left and right HG. The M50 to the first click was identified as the first upward oriented dipole before the M100 in the time interval 35-80 ms. The dipole with the best goodness of fit within a 6-ms interval around the peak of the M50-wave was considered as location of the M50 generator. Only dipole fits with goodness of fit greater than $80 \%$ were considered as reliable. Dipole localizations and orientations were determined for ACPC-aligned individual MRI datasets.

\subsection{Coordinate transformations}

FMRIB Software Library (FSL) (FMRIB Analysis Group, Oxford, UK) (Smith et al., 2004; Woolrich et al., 2009) was used for coordinate transformations. The new coordinate system based on the individual Heschl's Gyrus will be labeled HG-coordinate system. Dipole localizations obtained from Curry V.6 software were superimposed to the transformed MRI datasets and localizations were read out in millimeter-coordinates for further transformations.

The subsequent transformation into the HG-coordinate system was constructed as follows.

The starting point for the transformation in HG-coordinate system is an ACPC-aligned MRI dataset. Using the axial plane touching the superior edge of the AC and the inferior edge of the PC (XY-plane in an ACPC-based coordinate system) a first step to determine the HG's landmarks is to locate the most posterior point of the insula's gray matter at the border of the temporal lobe (Fig. 1, left).

Then, the sagittal plane is determined which is parallel to the mid-sagittal plane and cuts the posterior point selected before 


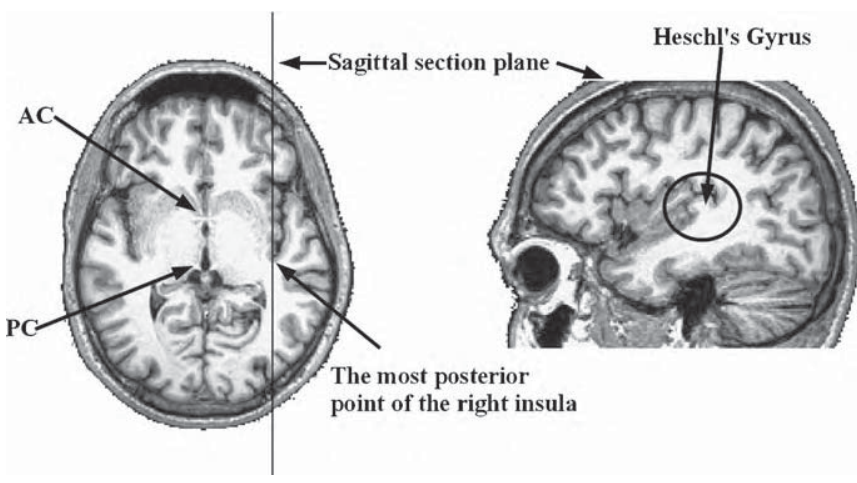

Fig. 1. Landmarks definition-step 1 . The first step for the coordinate system definition is to determine the position of the right HG with respect to the reference structures AC and PC.

(Fig. 1, left). In that sagittal plane the origin of the HG can be localized which extends transverse on the supratemporal plane (Fig. 1, right).

After the identification of the HG the most anterior and inferior (labeled HG1) as well as the most posterior and inferior (labeled HG2) points of the HG are located. Drawing first a line connecting the points HG1 and HG2 and another line perpendicular to the middle of that line allows to determine the point where this perpendicular line crosses the superior edge of the HG and label it as HG3. The middle of the distance between HG3 and the center of (HG1,HG2), labeled HG4, is defined as the origin of the new HG-based coordinate system (Fig. 2, left).

Furthermore the most distant point with respect to HG4 on the most anterior convolution of the right HG is determined as HG5 (Fig. 2, right). Thereby, the landmarks needed for the definition of the new coordinate system are completed.

The next step accomplishes the construction of the HGcoordinate system. Starting from the plane formed by the three points HG1, HG2 and HG3, a line parallel to the (HG1,HG2)-line was drawn to cross the HG4 point. The intersection of that line with the anterior edge of the HG was labeled temporary point one (T1) and the intersection with the posterior edge with temporary point two (T2) (Fig. 3, left).

In the plane created by $\mathrm{T} 1, \mathrm{~T} 2$ and HG5, a line perpendicular to the line (HG4, HG5) that crossed the HG4 (Fig. 3, right) is used as a $Y$-axis with a positive direction towards left anterior. The line connecting HG4 with HG5 was chosen as an $X$-axis with positive direction towards HG5. The Z-axis is chosen to be perpendicular to the $X Y$-plane with positive superior direction (right-handed coordinate system). The coordinate system for the left HG was chosen to be left handed with $X$-axis (HG4,HG5), $Y$-axis pointing in
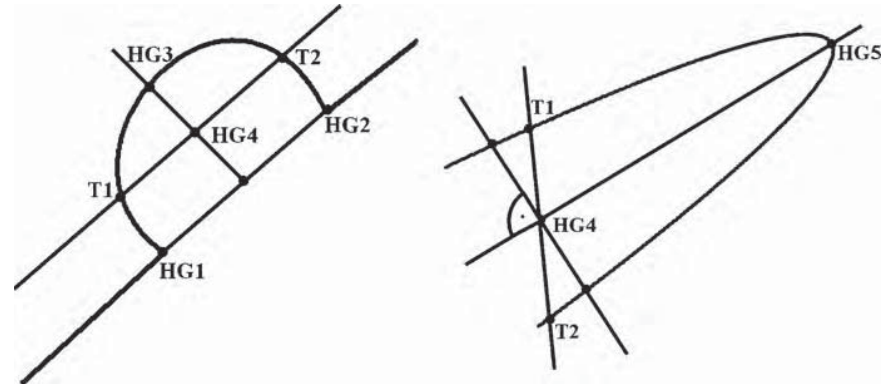

Fig. 3. Heschl's Gyrus coordinate system construction-Part 1. Step one (left) and step two (right) for the construction of the HG-coordinate system. With these steps the $X Y$-plane of the new coordinate system is completely defined.

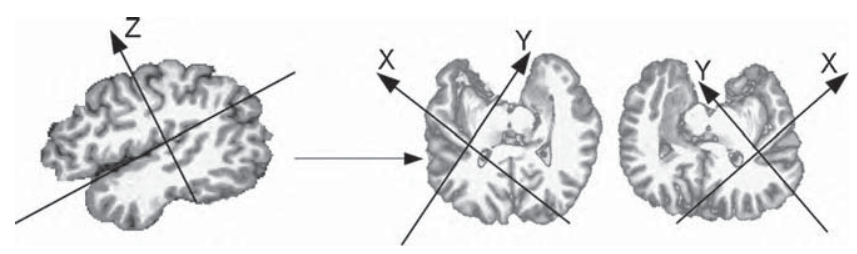

Fig. 4. Heschl's Gyrus coordinate system construction-Part 2. The construction of the HG-coordinate system is complete. For the left $\mathrm{HG}$ the $X$-axis is pointing left anterior, the $Y$-axis right anterior and $Z$-axis in superior direction (left handed coordinate system). The coordinate system for the right HG have switched $X$ and $Y$ axes, hence, right-handed coordinate system.

right-anterior direction and Z-axis with positive superior direction (Fig. 4).

An additional step for taking the length of the individual HGs into account is to normalize with respect to the distance between the points HG4 and HG5. Dividing the $X$-axis values with that normalization factor results in a HG's length that is equal one for all subjects and hemispheres. In order to get the length again in millimeter one has to multiply the $X$-axis values with a reference value. Possible choices for such a reference value may be the mean value over all subjects and hemispheres or the mean length over all subjects calculated separately for each hemisphere. In this paper the first method is used-the average over subjects and hemispheres (mean length $36 \mathrm{~mm}$ ).

The hypothesis of reducing the interindividual structural variability by the HG transformation (HGT) was evaluated by comparing the distribution of a points chosen by a scientist naive to the HGT, separately for each dataset, one point in the middle of the HG for each HG using the individual's MRI dataset in ACPC-coordinate system. Then, both methods, HGT and FNIRT (FMRIB's Nonlinear Image Registration Tool), were used with these
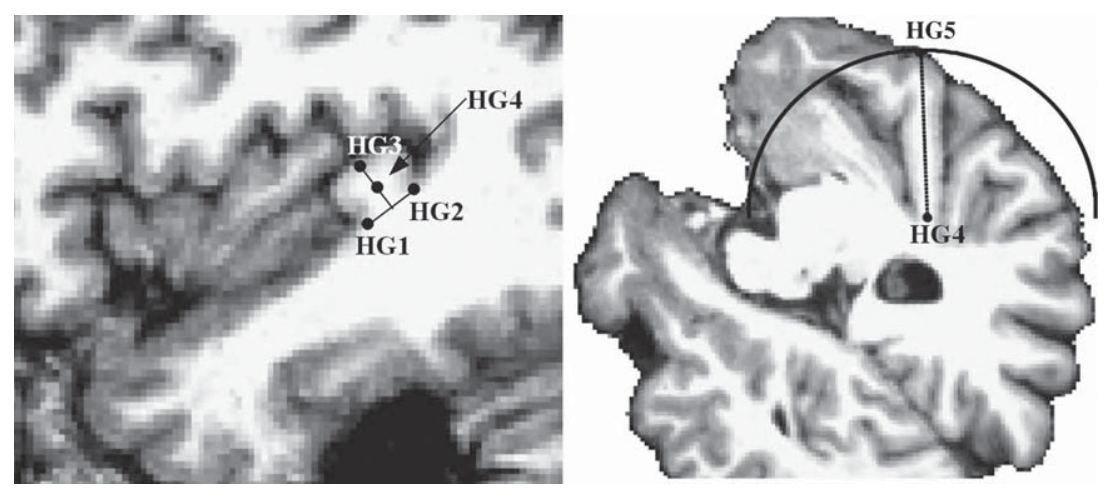

Fig. 2. Landmarks definition-step 2. HG4 has been chosen as an origin for the new coordinate system. HG5 was determined as the most right-lateral point on the anterior convolution of HG. 


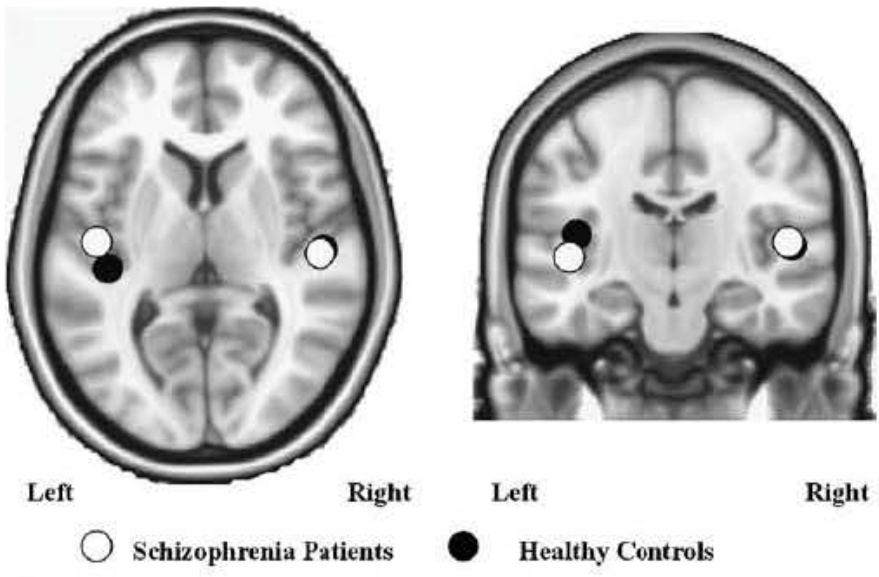

Fig. 5. Average M50 dipole locations for both groups with FNIRT. Mean values of M50 dipole localizations for schizophrenia patients and healthy controls after FNIRT registration.

points with the standard deviation(SD) in three-dimensional space $\left(\sqrt{\mathrm{SD}_{x}^{2}+\mathrm{SD}_{y}^{2}+\mathrm{SD}_{z}^{2}}\right.$, where $\mathrm{SD}_{x}, \mathrm{SD}_{y}, \mathrm{SD}_{z}$ are the standard deviations in one dimensional space for the axes $X, Y$ and $Z$, respectively) for each hemisphere as dispersion measure after the transformations.

Asymmetry of source localization between both groups in all coordinate systems was analyzed with a repeated measures ANOVA using group (controls, patients) as a between-group and hemisphere (left, right) as a within-group factor. Validity of the HGT for functional measures was demonstrated by comparing the anterior-posterior asymmetry of the M50 response between healthy control subjects and schizophrenia patients (Fig. 5).

At last error propagation for HGT and FNIRT is considered. A point in the middle of the right HG was chosen in a single subject's coordinate system based on the anterior and posterior commissures (ACPC-coordinate system) and transformed using both-HGT and FNIRT. Then, the same point was shifted with $1 \mathrm{~mm}$ towards
Table 1

Standard deviation comparison between HGT and FNIRT for the points chosen by a naive rater.

\begin{tabular}{llll}
\hline \multicolumn{4}{l}{ Standard deviation of the chosen points (in mm) } \\
\hline Transformation & ACPC & HGT & FNIRT \\
\hline Left & 6.77 & 3.83 & 3.84 \\
Right & 6.08 & 2.29 & 2.59 \\
\hline
\end{tabular}

posterior direction (in ACPC-aligned MRI) and transformed again with both methods. The resulting points were compared with the original point by measuring the Euclidean distance between the new shifted points to the original ones (absolute error). Those procedure of shifting was repeated with 2,3 , and $4 \mathrm{~mm}$, up to $15 \mathrm{~mm}$ from the original point.

\section{Results}

The SD for the points labeling the middle of each HG do not differ between both methods-HGT and FNIRT. For the left hemisphere the values are almost identical for both methods (Table 1,3rd row). For the right hemisphere results differ by $0.3 \mathrm{~mm}$ (advantage for the HGT) (Table 1, 4th row), whereas both methods have a lower SD than the ACPC-based alignment (Table 1, 2nd column).

Further results about the characteristics of the HGT were demonstrated by asymmetry differences between schizophrenia patients and healthy controls. In an ACPC-based coordinate system a significant interaction hemisphere $\times$ group $(F(1,18)=5.76$, $p<0.05)$ confirmed that M50 was localized more anterior in the right than in the left hemisphere in controls (post hoc Fisher LSD-test $p=0.04$ ), whereas the patient group did not show hemispheric differences in M50-localization (Table 2, 2nd column). When transformed into the HG-coordinate system (Fig. 6), a significant interaction hemisphere $\times$ group $(F(1,18)=8.23, p<0.02)$ confirmed not only hemispheric asymmetry in controls (post hoc Fisher LSD-test: $p<0.03$ ) but also indicated more posterior M50localization in the left hemisphere in controls compared to patients $(p<0.02$ ) (Table 2, 3rd column).

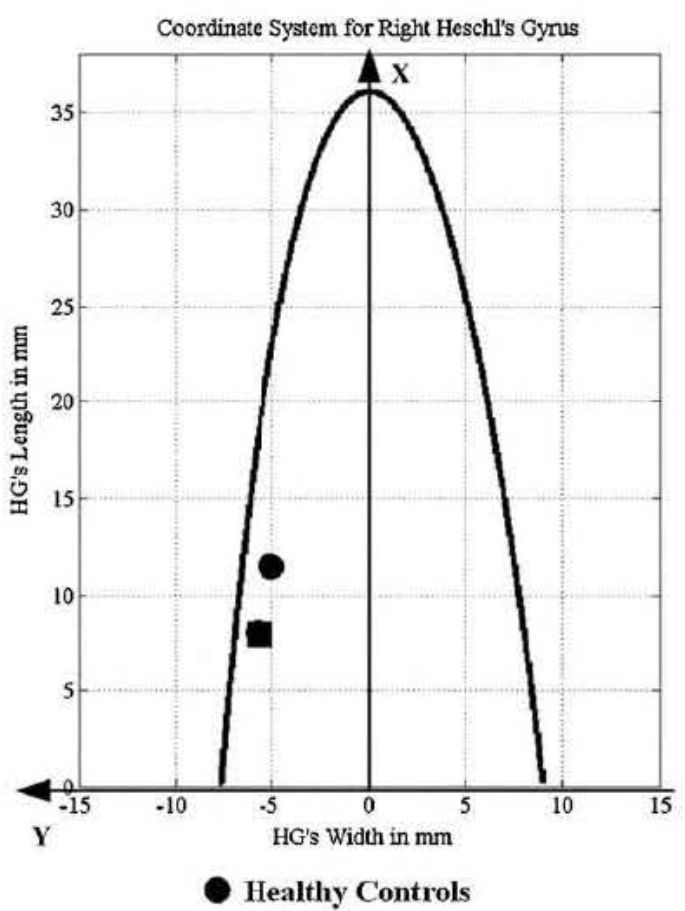

Fig. 6. Average M50 dipole locations for both groups with HGT. Mean values of dipole localizations in HG-coordinate system for schizophrenia patients and healthy controls. 
Table 2

p-values for the asymmetry in the different coordinate systems.

\begin{tabular}{llll}
\hline \multicolumn{4}{l}{ Statistical group differences in the dipole positions ( $p$-values) } \\
\hline Transformation & ACPC & HGT & FNIRT \\
\hline Interaction & 0.03 & 0.01 & 0.01 \\
Patients vs. controls left & 0.11 & 0.02 & 0.03 \\
Controls left vs. controls right & 0.04 & 0.02 & 0.01 \\
\hline
\end{tabular}

Table 3

Standard deviation for the dipole localizations for both methods-HGT and FNIRT.

\begin{tabular}{lll}
\hline \multicolumn{2}{l}{ Standard deviation of dipole localizations (in mm) } \\
\hline Transformation & HG & FNIRT \\
\hline Left & 14.67 & 16.24 \\
Right & 12.11 & 12.56 \\
\hline
\end{tabular}

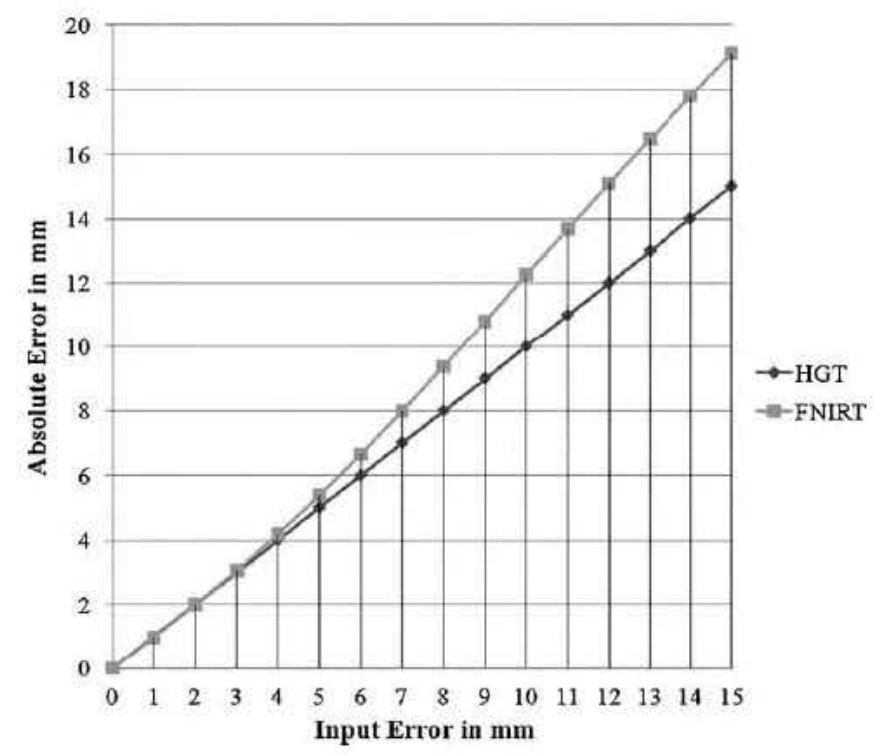

Fig. 7. Error propagation. Absolute error for HGT and FNIRT.

The interaction was confirmed also for FNIRT $(F(1,18)=7.47$, $p<0.02$ ). For between-group differences in the left hemisphere (post hoc Fisher LSD-test: $p<0.03$ ) FNIRT resulted in higher $p$-value compared to HGT(Table 2, 4th row). FNIRT yielded better score than HGT in the between hemispheres comparison for the controls (post hoc Fisher LSD-test: $p<0.01$ ) (Table 2, 5th row).

The SD of the dipole localizations for both hemispheres suggests better results using HGT compared to FNIRT. The SD for the left hemisphere in HGT was $1.5 \mathrm{~mm}$ smaller than the same in FNIRT, for the right hemisphere HGT was better by $0.4 \mathrm{~mm}$ than FNIRT (Table 3).

In addition both methods differ in sensitivity for error afflicted points (such points are e.g., dipole localizations). For HGT linear relationship between the input error and the absolute error was assessed in contrast to FNIRT, which provides a nonlinear increase for points with more than $3 \mathrm{~mm}$ input error (Fig. 7).

\section{Discussion}

Development of a HG-coordinate transformation aimed to (a) account for interindividual variability of structure, (b) relate functional activity to a reference structure and (c) improve the precision of functional measures in the primary auditory cortex for group comparisons. Results indicate that the HG-coordinate transformation met the first aim. The dispersion in the HG-coordinate system was considerably smaller than the one in the ACPC-coordinate system (Table 1, 2nd column). Comparison with FNIRT supported the accuracy performance of the HG-coordinate system transformation (Table 1, 3rd and 4th columns).

Moreover, the HG-coordinate system transformation allows the assignment of functional measures to a specific structure. Being entirely based on the position and orientation of the HG in the individual brain, localization of functional measures (such as M50) in the HG-coordinate system can be assumed to reflect the function of this reference structure. The comparison of M50-asymmetry between controls and schizophrenia patients in ACPC-coordinate system did not allow a conclusion whether the differences in asymmetry resulted from structural or functional differences. Group-specific auditory source localization relative to the anterior commissure does not allow to assume activity in the HG. The asymmetry of M50-localization may have resulted from structural asymmetry, since the right $\mathrm{HG}$ in patients is located more posterior relative to the AC compared to the right HG in controls. As a consequence the dipoles measuring their functional activity may have varied. Differences in the auditory cortical activity map can only be assumed after transformation of functional source localizations into a coordinate system based on the brain structure contributing to that function. In the present study comparison of asymmetry of activity localizations after transformation into the HG-coordinate system confirmed significant differences between healthy controls and schizophrenia patients. This indicates that the asymmetry differences may depend not only on the anterior-posterior asymmetry of the HG. They also show differences in the functional map. Moreover, transformation into the HG-coordinate system provided information not manifest in the ACPC-coordinate system, in that healthy subjects exhibit more posterior sources in the left hemisphere than patients. It should be noted, however, that structural differences between schizophrenic patients and healthy control participants may exist, but are transformed away by the HGT. Thus, the finding that functional differences exist between patient groups does not rule out anatomical group differences. The importance of the current method lies in its ability to differentiate between anatomical and functional group differences.

Another advantage of the HGT compared to FNIRT can be recognized by looking at the absolute error plot (Fig. 7) and the SD for the dipole localizations (Table 3). All nonlinear approaches augment the input error if it exceeds a certain amplitude (for FNIRT this limit is $3 \mathrm{~mm}$ for a concrete MRI dataset). The same phenomenon is evident in the standard deviation of the dipole localizations in the left hemisphere. A standard deviation value larger than $10 \mathrm{~mm}$ caused a greater dispersion for the nonlinear approach (here FNIRT) compared to HGT. Since single equivalent current dipole localizations are always afflicted with errors due to inverse problem uncertainty, low signal to noise ratio or subjects' movement during the measurement, it is likely that these errors increase additionally even more when using nonlinear deformation methods.

Finally, the practicality of the method should be commented on. As long as the MRI images are of good quality, identification of the points needed for the HGT is straight forward after a short amount of practice.

In sum, the present results clearly indicate the advantage of referring functional measures to a coordinate system that is based on the brain structure corresponding for that function. Considering structural variability increases sensitivity and, hence, validity of functional analysis. Whereas this conclusion refers to functional activity in the primary auditory cortex and a HG-based coordinate system, the present method and results may encourage the development of similar coordinate system transformation for other cortical structures. 


\section{Acknowledgment}

Research was supported by the Deutsche Forschungsgemeinschaft (Ro 805/14).

\section{References}

Adler LE, Pachtman E, Franks RD, Pecevich M, Waldo MC, Freedman R. Neurophysiological evidence for a defect in neuronal mechanisms involved in sensory gating in schizophrenia. Biological Psychiatry 1982;17(6):639-54.

Barta PE, Pearlson GD, Brill 2nd LB, Royall R, McGilchrist IK, Pulver AE, et al. Planum temporale asymmetry reversal in schizophrenia: replication and relationship to gray matter abnormalities. American Journal of Psychiatry 1997;154(5):661-7.

Dickson J, Drury H, Van Essen DC. 'The surface management system' (SuMS) database: a surface-based database to aid cortical surface reconstruction, visualization and analysis. Philosophical Transactions of the Royal Society of London Series B, Biological Sciences 2001;356(1412):1277-92.

Engel K, Tönnies K, Brechmann A. Parcellation of the auditory cortex into landmarkrelated regions of interest. In: Proceedings of the 13th international conference on computer analysis of images and patterns. Münster, Germany: SpringerVerlag; 2009. p. 631-8.

Evans AC, Collins DL, Mills SR, Brown ED, Kelly RL, Peters TM. 3D statistical neuroanatomical models from 305 MRI volumes. Proceedings of the IEEE Nuclear Science and Symposium Medical Imaging 1993;3(1-3):1813-7.

Fischl B, Sereno MI, Tootell RB, Dale AM. High-resolution intersubject averaging and a coordinate system for the cortical surface. Human Brain Mapping 1999;8(4):272-84

Friston KJ, Ashburner J, Frith CD, Poline J, Heather JD, Frackowiak RSJ. Spatial registration and normalization of images. Human Brain Mapping 1995;3(3):165-89.

Hirayasu Y, McCarley RW, Salisbury DF, Tanaka S, Kwon JS, Frumin M, et al. Planum temporale and Heschl Gyrus volume reduction in schizophrenia: a magnetic resonance imaging study of first-episode patients. Archives of General Psychiatry 2000;57(7):692-9.

Kang X, Bertrand O, Alho K, Yund EW, Herron TJ, Woods DL. Local landmark-based mapping of human auditory cortex. NeuroImage 2004;22(4):1657-70.
Kasai K, Shenton ME, Salisbury DF, Hirayasu Y, Onitsuka T, Spencer MH, et al. Progressive decrease of left Heschl Gyrus and planum temporale gray matter volume in first-episode schizophrenia: a longitudinal magnetic resonance imaging study. Archives of General Psychiatry 2003;60(8):766-75.

Kim J, Crespo-Facorro B, Andreasen NC, O'Leary DS, Zhang B, Harris G, et al. An MRI-based parcellation method for the temporal lobe. Neurolmage 2000;11(4):271-88.

Petty R, Barta P, Pearlson G, McGilchrist I, Lewis R, Tien A, et al. Reversal of asymmetry of the planum temporale in schizophrenia. American Journal of Psychiatry 1995;152(5):715-21.

Rossi A, Serio A, Stratta P, Petruzzi C, Schiazza G, Mancini F, et al. Planum temporale asymmetry and thought disorder in schizophrenia. Schizophrenia Research 1994;12(1):1-8.

Rossi A, Stratta P, Mattei P, Cupillari M, Bozzao A, Gallucci M, et al. Planum temporale in schizophrenia: a magnetic resonance study. Schizophrenia Research 1992;7(1):19-22.

Smith SM, Jenkinson M, Woolrich MW, Beckmann CF, Behrens TE, Johansen-Berg H, et al. Advances in functional and structural MR image analysis and implementation as FSL. NeuroImage 2004;23(Suppl. 1):208-19.

Tahmasebi AM, Abolmaesumi P, Zheng ZZ, Munhall KG, Johnsrude IS. Reducing intersubject anatomical variation: effect of normalization method on sensitivity of functional magnetic resonance imaging data analysis in auditory cortex and the superior temporal region. Neurolmage 2009;47(4):1522-31.

Talairach J, Tournoux P. Co-planar stereotaxic atlas of the human brain. New York: Thieme Medical; 1988

Thompson P, Toga AW. A surface-based technique for warping three-dimensional images of the brain. IEEE Transactions on Medical Imaging 1996;15(4): 402-17.

Van Essen DC, Drury HA. Structural and functional analyses of human cerebral cortex using a surface-based atlas. Journal of Neuroscience 1997;17(18):7079-102.

Van Essen DC, Drury HA, Joshi S, Miller MI. Functional and structural mapping of human cerebral cortex: solutions are in the surfaces. Proceedings of the National Academy of Sciences of the United States of America 1998;95(3):788-95.

Viceic D, Campos R, Fornari E, Spierer L, Meuli R, Clarke S, et al. Local landmark-based registration for fMRI group studies of nonprimary auditory cortex. NeuroImage 2009;44(1):145-53.

Woolrich MW, Jbabdi S, Patenaude B, Chappell M, Makni S, Behrens T, et al. Bayesian analysis of neuroimaging data in FSL. Neurolmage 2009;45(1 Suppl. 1):173-86. 